\title{
Radiation Therapy and Non-Hodgkin Lymphoma of the Extremity. Case Reports: Strategy for Treatment Planning and Therapy Implementation
}

\author{
Kevin 0'Connor ${ }^{1}$ | James Shen ${ }^{1}$ | Ameer Elaimy ${ }^{1}$ | Rebecca Wang ${ }^{1}$ | Linda Ding ${ }^{1}$ | Harry Bushe ${ }^{1}$ | Carla \\ Bradford $^{1} \mid$ I-Lin Kuo ${ }^{1}$ | Yankhua Fan ${ }^{1}$ | Abdulnasser Khalifeh ${ }^{1}$ | Fenhong Liu ${ }^{1}$ | Suhong Yu ${ }^{1}$ | Jonathan \\ Saleeby $^{1}$ | Samantha Boudreau ${ }^{1}$ | Erica Smith ${ }^{1}$ | Paul Rava ${ }^{1}$ | Shirin Sioshansi ${ }^{1}$ | Eric Ko ${ }^{1}$ | Daniel Han ${ }^{1}$ | M.

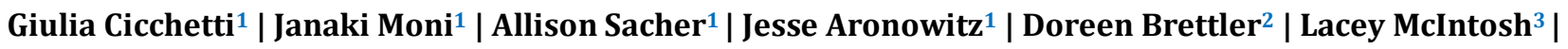 \\ Bruce Woda $^{4}$ | Jacob Bledsoe ${ }^{4}$ | Mathew Most ${ }^{5}$ | Maryann Bishop-Jodoin ${ }^{1}$ | Thomas J FitzGerald ${ }^{1}$
}

*Correspondence: Thomas J FitzGerald

Address: ${ }^{1}$ Departments of Radiation Oncology, University of Massachusetts Medical School, Worcester, MA. 01655, USA; ${ }^{2}$ Department of Medicine, University of Massachusetts Medical School, Worcester, MA. 01655, USA; ${ }^{3}$ Department of Radiology, University of Massachusetts Medical School, Worcester, MA. 01655, USA; ${ }^{4}$ Department of Pathology, University of Massachusetts Medical School, Worcester, MA. 01655, USA; ${ }^{5}$ Department of Orthopedics, University of Massachusetts Medical School, Worcester, MA. 01655, USA

e-mail $\bowtie:$ TJ.Fitzgerald@umassmemorial.org

Received: 30 December 2020; Accepted: 08 January 2021

Copyright: (C) 2021 O'Connor K. This is an open-access article distributed under the terms of the Creative Commons Attribution License, which permits unrestricted use, distribution, and reproduction in any medium, provided that the original work is properly cited.

\section{ABSTRACT}

Radiation therapy delivered with definitive intent is challenging in the treatment of malignancies involving the extremities. Often, the target volume of interest is circumferential in the extremity compartment and sparing normal tissue and providing conformal avoidance to bone/soft tissue is difficult to achieve. In this manuscript we review the use of volumetric modulated arc therapy (VMAT) to provide concurrent and simultaneous radiation treatment to tumors in both anterior and posterior compartments including the popliteal fossa with appropriate sparing of normal tissue for lymphatic drainage. The use of advanced therapy technology can make a significant difference in the comprehensive management of patients with both epithelial and non-epithelial non-Hodgkin lymphoma lesions in the extremities.

Keywords: Radiation Therapy, Non-Hodgkin Lymphoma, Therapy Implementation

\section{Introduction}

Radiation therapy management with definitive intent for patients with soft tissue and bone disease in the extremities is challenging for providers. Therapy planning strategies need to include immobilization of the extremity as well as a strategy for sparing normal tissue for appropriate extremity lymphatic drainage to ensure an optimal functional outcome. From a historical perspective, definitive extremity care is thought to be optimally managed with rigid immobilization for daily treatment reproducibility and static fields with light field validation to provide reassurance that normal tissue is 
being spared from therapy for appropriate lymphatic drainage of tissues distal to the intended target (Zheng et al., 2012).

Non-Hodgkin lymphoma incidence is increasing at all age levels. We continue to observe a significant increase in patients particularly in older populations with disease exhibiting intermediate and high-risk features requiring vigorous application of both systemic and local therapy. Competing medical comorbidities can influence the application and duration of systemic management including targeted and immune driven therapy. Therefore, radiation therapy is often applied at various timepoints during the care of the patient to all body regions, including indications when systemic therapy options are limited due to the medical status of the patient and unintentional potential for therapy-associated toxicity with systemic management.

In this paper we review two patients with non-Hodgkin lymphoma of the extremity with complex target geometries and review how VMAT can be applied with optical tracking systems deployed to successfully reproduce volumes for treatment without the need for rigid devices which can be difficult to build and reproduce treatment accurately daily. The volumes of therapy are especially challenging as targets in both the anterior and posterior aspect of the lower extremity required treatment with full dose. We review the strategy for conformal avoidance of key structures including placing dose gradients across various points of joint spaces as well as spare a strip of normal tissue to preserve lymphatic drainage.

\section{Clinical Presentation}

Selected patients with extremity non-Hodgkin lymphoma treated by our department were reviewed for radiation plan development and stability of daily treatment execution. Patients completed the planned course of radiation therapy. Two patients were chosen for the focus of this manuscript. Both required radiation therapy to be delivered across normal tissue including the knee joint. The ability of volume modulated arc therapy to limit dose across normal tissue structures was compared to traditional conventional therapy plans. This was reviewed for statistical significance with a two sample $\mathrm{T}$ test designed to evaluate the significance of volume reduction in normal tissue dose between traditional anterior-poster therapy and therapy applied with VMAT.

\section{Patient One}

This patient had multiple recurrences of diffuse large B-cell non-Hodgkin lymphoma at the time he was treated to the right lower extremity including multiple previous regimens of chemoimmunotherapy. The site of origin of disease was thought to be the gastric fundus. Stage 4 disease was 
established at presentation with disease located in multiple soft tissue regions, lymph nodes, and bone without involvement of hepatic or pulmonary parenchyma. As part of consolidation management after chemotherapy, he was treated with radiation therapy to multiple sites of disease including the right axilla and two dermal sites of disease in the head/neck. At the time of therapy failure, the patient developed multiple sites of disease in the dermal surfaces of the anterior compartment of the right lower extremity along the tibial plateau. Clinical examination and positron emission tomography confirmed popliteal adenopathy creating a challenge in treatment both anterior and posterior disease. Volume modulated arc management permitted target coverage of disease requiring placing dose gradients across multiple areas of normal tissue for lymphatic drainage including the joint space at the level of the knee. The use of kilo voltage $(\mathrm{kV})$ on line image guidance coupled with periodic use of cone beam computer tomography and optical tracking systems for daily positioning and alignment greatly facilitated patient care as rigid structures for immobilization were not required. This greatly facilitated patient comfort and the speed of treatment as therapy. Traditional static field therapy would have taken much longer to complete coupled with the potential decreased security imposed by intra-fractional motion. In total, more than 30 minutes of time would be required for therapy including validation of patient set up. The VMAT plan with daily reproducibility took a few minutes to complete post image validation which likewise contributed to the ease of therapy set up and successful treatment execution. Fig. 1 represents an axial view of the treatment plan which was reproduced daily with $\mathrm{kV}$ imaging and monitored with optical tracking to ensure stability in the set up during therapy. The volume modulated arc plan outperformed the traditional plan with respect to sparing normal tissue including the knee joint and was statistically significant compared to a traditional AP-PA or three-dimensional plan.

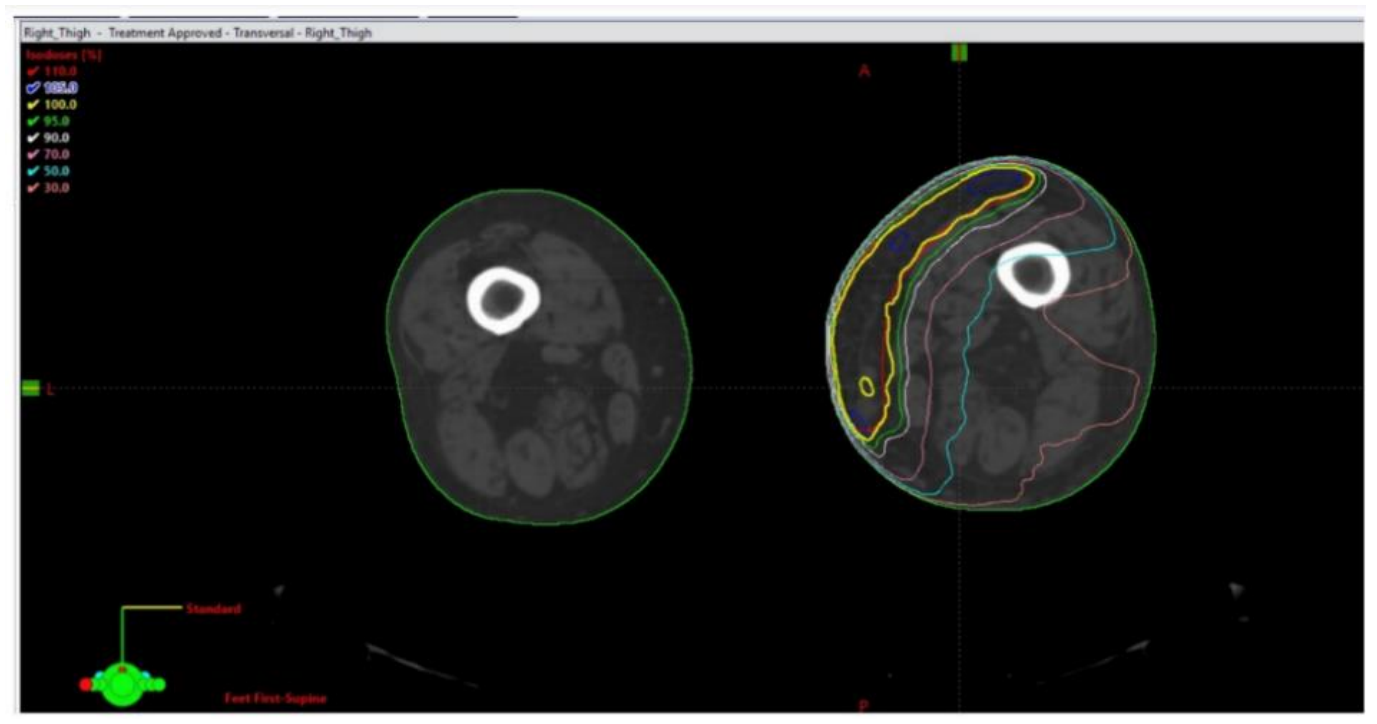

Figure 1: Axial view of the treatment plan which was reproduced daily with $\mathrm{kV}$ imaging and monitored with optical tracking to ensure stability in the set up during therapy, Courtesy of the Department of Radiation Oncology, University of Massachusetts Medical School. 


\section{Patient Two}

This patient had a stage 2AE diffuse large B-cell non-Hodgkin lymphoma of the lower extremity whose disease at presentation involved the tibia by compression with near circumferential disease involving the left lower extremity including popliteal adenopathy. Inguinal/femoral adenopathy at presentation was treated with a discontinuous plan. Fig. 2 represents the plan using volume modulated arcs which was designed to treat all sites of disease including the circumferential tumor target, popliteal region, and component of the tibia initially involved with tumor. The patient was treated with optical tracking without rigid immobilization which provided security in daily positioning. As in patient 1 , the volume modulated arc plan out performed the traditional plan with respect to normal tissue including the knee joint and was statistically significant compared to alternate three dimensional plans.

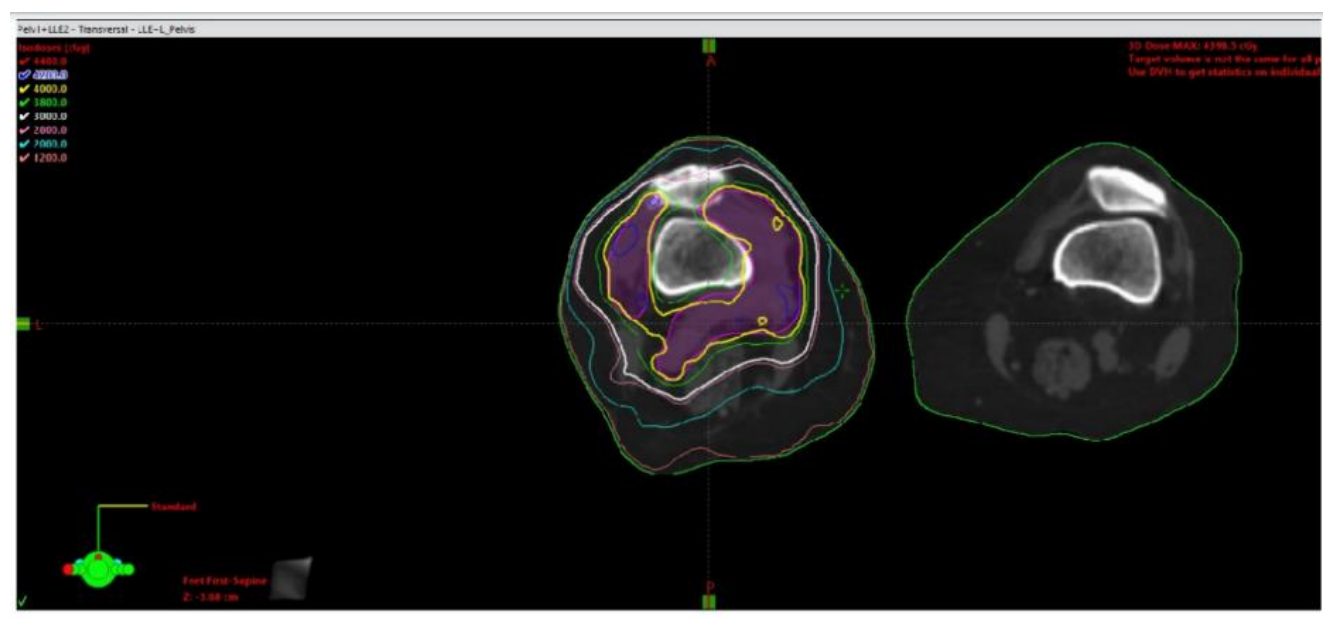

Figure 2: The plan using volume modulated arcs which was designed to treat all sites of disease including the circumferential tumor target, popliteal region, and component of the tibia initially involved with tumor. Courtesy of the Department of Radiation Oncology, University of Massachusetts Medical School.

\section{Discussion}

Radiation therapy is often delivered to extremities in a palliative effort with planning designed for patient comfort and rapid workflow, often with non-volumetric treatment plans. In contrast, when therapy is delivered with definitive intent, multiple issues need to be considered to provide optimal management for patient care (Tiwana et al., 2016). There needs to be a strategy for protection of normal tissue for lymphatic flow (Christiansen et al., 2016; Ishii et al., 2017; Gonzalez-Vela et al., 2008; Dargent et al., 2005; Persky et al., 2008; Torres-Paoli and Sanchez, 2000). Therapy delivered to skin folds and joint spaces must be carefully designed for dose uniformity and prevention of late effects to tissue of limited self-renewal capacity. We are learning that therapy across bony structures in adults can cause insufficiency fractures in weight bearing regions, therefore it is important to ensure radiation therapy 
dose gradients are placed across bony segments when possible and "hot spots" are avoided in bone (Tokumaru et al., 2012).

It is difficult to design radiation therapy to both anterior and posterior structures in an extremity and spare normal tissue for optimal orthopedic functional outcome, especially when therapy crosses joint spaces (Zheng et al., 2012). Table 1 shows the volume of normal tissue excluded from high dose when highly conformal plans can be applied to complex geometries in both cases. For these cases, dose to joint spaces was considerably improved applying VMAT for conformal avoidance. When disease crosses joint spaces and involves structures in different planes, only advanced technology planning can achieve the objective of disease management coupled with normal tissue exclusion. Intensity modulation including the use of VMAT permits full dose therapy to targets and supports normal tissue sparing. The planning of care requires skill in the physics of therapy plan design, however of equal challenge is the daily reproducibility of therapy. Historically, most radiation oncology plans were designed with rigid casting. Although there were strengths in positioning stability to this approach, the extremity was not always easily placed into the structure and air gaps within the structure coupled with tissue equivalent composition of the cast created dose asymmetries including augmenting dose to superficial structures. This would compound management during radiation therapy and if therapy was applied with pre-operative intent to high dose, wound healing after surgery could be compromised. Therefore, technique and application of technique can significantly influence outcome to tumor control and long-term functional outcome.

Table 1: Volume of normal tissue excluded from high dose when extremely conformal plans, using VMAT, can be applied to complex geometries in two cases. The volume treated is significant at $\mathrm{P}<0.05$.

\begin{tabular}{|c|c|c|c|}
\hline & $\begin{array}{l}\text { Plan Type: } \\
\text { anterior-posterior (AP)/ posterior- } \\
\text { anterior (PA) }\end{array}$ & $\mathrm{V} 100 \%$ (cc) & $\begin{array}{l}\text { Normal Tissue Receiving } 100 \% \\
\text { Treatment Dose (cc) }\end{array}$ \\
\hline \multirow[t]{2}{*}{ Patient One } & AP-PA & 1156.5 & 617.4 \\
\hline & VMAT & 574.9 & 35.8 \\
\hline \multirow{2}{*}{$\begin{array}{l}\text { Patient Two } \\
\text { Left lower extremity }\end{array}$} & AP-PA & 1970.5 & 1616.3 \\
\hline & VMAT & 458.2 & 104.0 \\
\hline \multirow{2}{*}{$\begin{array}{l}\text { Patient Two } \\
\text { Left pelvis }\end{array}$} & AP-PA & 1317.7 & 1137.9 \\
\hline & VMAT & 206.5 & 26.7 \\
\hline
\end{tabular}

In this paper we review two cases of non-Hodgkin lymphoma involving the lower extremity. Both cases required skill in planning and the use of volumetric modulation applied to the target permitted appropriate radiation dose to target with exceptional normal tissue sparing. Patient One required a field length of $>40 \mathrm{~cm}$, therefore dynamic tools were employed to accommodate his care into a single plan and the second patient presented with near circumferential target volume making normal tissue sparing very challenging. Optical tracking provided a nimble and reproducible tool to confirm the appropriate 
application of daily therapy without the need for rigid immobilization. The daily therapy set up in a nimble manner and the use of optical tracking with VMAT significantly reduced treatment time from more than 30 minutes to less than 10 minutes serving to enhance confidence in the daily set up. This is important as we titrate therapy volumes because we need security that the daily set up is precise as volume titration leaves less room for set up variability, especially in cases involving the extremity in which modest motion can create clinically significant changes including unintended treatment of tissue and under dose of intended target.

Modern radiation therapy has moved into a very good position to optimize care of complex geometries in multiple target locations including the extremities. As we become more skilled at applying these tools, patient outcomes as measured by functional outcomes and patient satisfaction will continue to improve.

\section{References}

Christiansen S, Cohen P, Broome C. A case series of non-Hodgkin's lymphoma recurrence in lower extremities mimicking deep vein thrombosis. Blood 2016; 128: 5344.

Dargent JL, Lespagnard L, Feoli F, Debusscher L, Greuse M, Bron D. De novo CD5-positive diffuse large B-cell lymphoma of the skin arising in chronic limb lymphedema. Leukemia \& Lymphoma 2005; 46: 775-780.

Gonzalez-Vela MC, Gonzalez-Lopez MA, Val-Bernal J, Fernandez-Llaca H. Cutaneous diffuse B cell lymphoma of the leg associated with chronic lymphedema. International Journal of Dermatology 2008; 47: 174-177.

Ishii N, Aoki M, Kishi K. Lymphedema of the lower extremities due to refractory malignant lymphoma treated by lymphaticovenous anastomosis. Plastic Reconstructive Surgery Global Open 2017; pp: e1475.

Persky DO, Unger JM, Spier CM, Stea B, LeBlanc M, McCarty MJ, Rimsa LM, Fisher RI, Miller TP, Southwest Oncology Group. Phase II study of rituximab plus three cycles of CHOP and involved-field radiotherapy for patients with limited-stage aggressive B-cell lymphoma: Southwest Oncology Group study 0014. Journal of Clinical Oncology 2008; 26: 2258-2263.

Tiwana M, Barnes M, Kiraly A, Olson R. Utilization of palliative radiotherapy for bone metastasis near end of life in a population-based cohort. BMC Palliative Care 2016; 15: 2.

Tokumaru S, Toita T, Oguchi M, Ohno T, Kato S, Niibe Y, Kazumoto T, Kodaira T, Kataoka M, Shikama N, Kenjo M, Yamauchi C, Suzuki O, Sakurai H, Teshima T, Kagami Y, Nakano T, Hiraoka M, Mitsuhashi N, Kudo S. Insufficiency fractures after pelvic radiation therapy for uterine cervical cancer: an analysis of subjects in a prospective multi-institutional trial and cooperative study of the Japan Radiation Oncology Group (JAROG) and Japanese Radiation Oncology Study Group (JROSG). International Journal of Radiation Oncology, Biology, Physics 2012; 84: 195-200.

Torres-Paoli D, Sanchez JL. Primary cutaneous B-cell lymphoma of the leg in a chronic lymphedematous extremity. American Journal of Dermatopathology 2000; 22: 257-260.

Zheng X, Dai T, Shu X, Pu Y, Feng G, Li X, Liao D, Du X. A new method of lower extremity immobilization in radiotherapy. Radiation Oncology 2012; 7: 27. 